\title{
Evidence of a Clear Atmosphere for WASP-62b: The Only Known Transiting Gas Giant in the JWST Continuous Viewing Zone
}

Alam, Munazza K.; López-Morales, Mercedes; MacDonald, Ryan J.; Nikolov, Nikolay; Kirk, James; Goyal, Jayesh M.; Sing, David K.; Wakeford, Hannah R.; Rathcke, Alexander D.; Deming, Drake L.

Total number of authors:

15

Published in:

Astrophysical Journal Letters

Link to article, DOI:

10.3847/2041-8213/abd18e

Publication date:

2021

Document Version

Publisher's PDF, also known as Version of record

Link back to DTU Orbit

Citation (APA):

Alam, M. K., López-Morales, M., MacDonald, R. J., Nikolov, N., Kirk, J., Goyal, J. M., Sing, D. K., Wakeford, H. R., Rathcke, A. D., Deming, D. L., Sanz-Forcada, J., Lewis, N. K., Barstow, J. K., Mikal-Evans, T., \& Buchhave L. A. (2021). Evidence of a Clear Atmosphere for WASP-62b: The Only Known Transiting Gas Giant in the JWST Continuous Viewing Zone. Astrophysical Journal Letters, 906(2), [L10]. https://doi.org/10.3847/2041$8213 / a b d 18 e$

\section{General rights}

Copyright and moral rights for the publications made accessible in the public portal are retained by the authors and/or other copyright owners and it is a condition of accessing publications that users recognise and abide by the legal requirements associated with these rights.

- Users may download and print one copy of any publication from the public portal for the purpose of private study or research.

- You may not further distribute the material or use it for any profit-making activity or commercial gain

- You may freely distribute the URL identifying the publication in the public portal 


\title{
Evidence of a Clear Atmosphere for WASP-62b: The Only Known Transiting Gas Giant in the JWST Continuous Viewing Zone
}

\author{
Munazza K. Alam ${ }^{1,12}$ (1) , Mercedes López-Morales ${ }^{1}$ (D), Ryan J. MacDonald ${ }^{2}$ (D) Nikolay Nikolov $^{3}$ (1), James Kirk $^{1}$ (1),

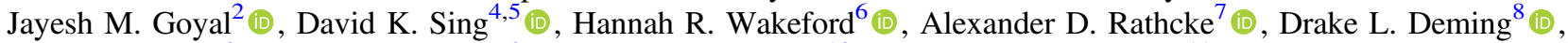 \\ Jorge Sanz-Forcada $^{9}$ (D), Nikole K. Lewis ${ }^{2}$ (D), Joanna K. Barstow ${ }^{10}$ (D), Thomas Mikal-Evans ${ }^{11}$ (iD), and Lars A. Buchhave ${ }^{7}$ (D) \\ ${ }^{1}$ Center for Astrophysics | Harvard \& Smithsonian, 60 Garden Street, Cambridge, MA 02138, USA; munazza.alam@cfa.harvard.edu \\ 2 Department of Astronomy and Carl Sagan Institute, Cornell University, Ithaca, NY 14853, USA \\ ${ }^{3}$ Space Telescope Science Institute, 3700 San Martin Drive, Baltimore, MD 21218, USA \\ ${ }^{4}$ Department of Earth \& Planetary Sciences, Johns Hopkins University, Baltimore, MD, USA \\ ${ }^{5}$ Department of Physics \& Astronomy, Johns Hopkins University, Baltimore, MD, USA \\ ${ }^{6}$ School of Physics, University of Bristol, HH Wills Physics Laboratory, Tyndall Avenue, Bristol BS8 1TL, UK \\ ${ }^{7}$ DTU Space, National Space Institute, Technical University of Denmark, Elecktrovej 328, DK-2800 Kgs. Lyngby, Denmark \\ ${ }^{8}$ Department of Astronomy, University of Maryland at College Park, College Park, MD 20742, USA \\ ${ }_{10}^{9}$ Centro de Astrobiología (CSIC-INTA), ESAC Campus, Villanueva de la Cañada, Madrid, Spain \\ ${ }^{10}$ School of Physical Sciences, The Open University, Walton Hall, Milton Keynes, MK7 6AA, UK \\ ${ }^{11}$ Kavli Institute for Astrophysics and Space Research, Massachusetts Institute of Technology, 77 Massachusetts Avenue, 37-241, Cambridge, MA 02139, USA \\ Received 2020 August 21; revised 2020 November 18; accepted 2020 November 22; published 2021 January 11
}

\begin{abstract}
Exoplanets with cloud-free, haze-free atmospheres at the pressures probed by transmission spectroscopy represent a valuable opportunity for detailed atmospheric characterization and precise chemical abundance constraints. We present the first optical to infrared $(0.3-5 \mu \mathrm{m})$ transmission spectrum of the hot Jupiter WASP-62b, measured with Hubble/STIS and Spitzer/IRAC. The spectrum is characterized by a $5.1 \sigma$ detection of Na I absorption at $0.59 \mu \mathrm{m}$, in which the pressurebroadened wings of the Na D-lines are observed from space for the first time. A spectral feature at $0.4 \mu \mathrm{m}$ is tentatively attributed to $\mathrm{SiH}$ at $2.1 \sigma$ confidence. Our retrieval analyses are consistent with a cloud-free atmosphere without significant contamination from stellar heterogeneities. We simulate James Webb Space Telescope (JWST) observations, for a combination of instrument modes, to assess the atmospheric characterization potential of WASP-62b. We demonstrate that JWST can conclusively detect $\mathrm{Na}, \mathrm{H}_{2} \mathrm{O}, \mathrm{FeH}, \mathrm{NH}_{3}, \mathrm{CO}, \mathrm{CO}_{2}, \mathrm{CH}_{4}$, and $\mathrm{SiH}$ within the scope of its Early Release Science (ERS) program. As the only transiting giant planet currently known in the JWST Continuous Viewing Zone, WASP-62b could prove a benchmark giant exoplanet for detailed atmospheric characterization in the James Webb era.
\end{abstract}

Unified Astronomy Thesaurus concepts: Exoplanet atmospheres (487); Planetary atmospheres (1244); Exoplanet atmospheric composition (2021)

\section{Introduction}

Close-in gas giant exoplanets that are cloud-free/haze-free in the observable atmosphere appear to be rare ( $<7 \%$ of cases), with the vast majority of hot Jupiters showing substantial opacity from condensation clouds and photochemical hazes (Wakeford et al. 2019). The few benchmark cases of clear atmospheres, such as WASP-96b (Nikolov et al. 2018) and WASP-39b (Fischer et al. 2016; Nikolov et al. 2016; Wakeford et al. 2018; Kirk et al. 2019), have produced some of the best constraints on atmospheric metallicities and abundances of $\mathrm{H}_{2} \mathrm{O}$ and $\mathrm{Na}$ to date (e.g., Welbanks et al. 2019). The rare class of cloud-free planets at the pressures probed via low-resolution transmission spectroscopy ( $\sim 10^{-3}-1$ bar for optical and infrared observations) permit precision measurements of atomic and molecular abundances, unhindered by cloud-composition degeneracies (e.g., Fraine et al. 2014; Kreidberg et al. 2014; Sing et al. 2016; Kilpatrick et al. 2018). Exoplanets with clear atmospheres therefore represent a valuable opportunity to unlock crucial insights into atmospheric chemistry and planetary formation history (e.g., Öberg et al. 2011; Mordasini et al. 2016).

The upcoming James Webb Space Telescope (JWST) will enable unprecedented detailed atmospheric characterization of exoplanets, exploring a wider wavelength range $(0.6-28.3 \mu \mathrm{m})$ than currently accessible with existing facilities

\footnotetext{
${ }^{12}$ National Science Foundation Graduate Research Fellow.
}

(Beichman et al. 2014). Its broad infrared wavelength coverage will allow precise molecular abundance constraints for many species, breaking degeneracies between parameters such as metallicity and the carbon-to-oxygen ratio. In preparation for the launch of JWST, the exoplanet community has invested a significant effort into identifying planets that are cloud-free/hazefree in the observable atmosphere for follow-up studies with JWST (see, e.g., Stevenson et al. 2016). One of the targets initially put forth is WASP-62b (Hellier et al. 2012), a $0.57 M_{\text {Jup }}, 1.39 R_{\text {Jup }}$ planet with $T_{\mathrm{eq}} \sim 1440 \mathrm{~K}$ orbiting a bright $(V=10.2) \mathrm{F} 7 \mathrm{~V}$ host star. Due to its fortuitous location in JWST's Continuous Viewing Zone (CVZ), i.e., near the south ecliptic pole, WASP-62b was identified as a potential target for the Transiting Exoplanet Community Early Release Science Program (ERS 1366; P.I.: N. Batalha) for JWST (Bean et al. 2018).

Although WASP-62b is one of the most favorable planets for JWST atmospheric studies, another target (WASP-79b, which is not located in JWST's CVZ) was chosen for the ERS program due to a lack of observational information about the atmospheric properties of WASP-62b. To date, WASP-62b is the only known transiting giant planet in the CVZ. Although TESS has found five giant planet candidates ${ }^{13}$ near the south

\footnotetext{
${ }^{13}$ Of the five TESS CVZ candidates, four are fainter than WASP-62 (with $V$-band magnitudes ranging from 11.3 to 12.4). The candidate brighter than WASP-62 at $V=8.6$ mag has a $\mathrm{V}$-shaped light curve and shows evidence of blending.
} 
ecliptic pole at the time of writing, none have been confirmed as planets (S. Quinn 2019, private communication). As a CVZ target, WASP-62b provides the opportunity to ensure that a suitable target is observable, regardless of any past or future JWST launch delays, with transit observations that can be flexibly scheduled and quickly executed.

In this Letter, we present the first optical transmission spectrum of the hot Jupiter WASP-62b. Our Hubble/STIS and Spitzer/IRAC observations demonstrate that this planet possesses a cloud-free terminator, rendering it a priority target for the JWST ERS Program. In what follows, we describe the data reduction and atmospheric retrieval of our new observations. We then discuss the implications of our atmospheric inferences, and provide testable predictions for infrared observations with JWST.

\section{Observations and Data Reduction}

We observed three transits of WASP-62b with STIS (Section 2.1) as part of the Hubble Panchromatic Comparative Exoplanetology Treasury (PanCET) program GO 14767 (PIs: Sing \& López-Morales). We observed two additional transits with Spitzer/IRAC (Section 2.2) in the $3.6 \mu \mathrm{m}$ and $4.5 \mu \mathrm{m}$ channels through GO 13044 (PI: Deming). Although Hubble/ WFC3 also observed one transit of this target for program GO 14767, we note that these observations suffered from guide star issues that render the data unreliable (Section 2.3).

\subsection{STIS}

The Hubble/STIS transit observations consist of lowresolution $(\sim 500)$ time series spectra collected on UT 2018 January 26 (visit 57) and UT 2018 May 12 (visit 58) with the G430L (2892-5700 ^) grism, and on UT 2017 November 21 (visit 59) with the G750L (5240-10270 ̊) grism. Each visit consisted of five consecutive 96 minutes orbits, during which 48 stellar spectra were obtained over exposure times of $253 \mathrm{~s}$. To decrease the readout times between exposures, we used a 128 pixel wide subarray. The data were taken with the $52 \times$ $2 \operatorname{arcsec}^{2}$ slit to minimize slit light losses. We note that both of the G430L visits suffered from guide star issues at the beginning of the observations, resulting in the loss of data collection during the first orbit (visit 57) or part of the first orbit (visit 58). From the subsequent exposures taken during these visits, however, we were able to extract good quality light curves with a median precision of $311 \mathrm{ppm}$.

We reduced the STIS spectra using the methods described in Alam et al. (2018, 2020). Briefly, we bias-, dark-, and flat-field corrected the raw 2D data frames using the CALSTIS pipeline (V 3.4). We corrected for cosmic-ray events using mediancombined difference images to flag and interpolate over bad pixels. We performed a 1D spectral extraction from the calibrated . flt files and extracted light curves using an aperture width of 13 pixels. From the x1d files, we obtained a wavelength solution by resampling all of the extracted spectra and cross-correlating them to a common rest frame. The crosscorrelation measures the shift of each stellar spectrum with respect to the first spectrum of the time series, so we resampled the spectra to align them and remove subpixel drifts associated with the different locations of the spacecraft on its orbit (Huitson et al. 2013).

\subsection{IRAC}

We observed two transits with Spitzer/IRAC on UT 2016 November 24 and UT 2016 December 7 in the $3.6 \mu \mathrm{m}$ and $4.5 \mu \mathrm{m}$ channels, respectively (Fazio et al. 2004; Werner et al. 2004). Each IRAC exposure was taken over integration times of $2 \mathrm{~s}$, resulting in 20,159 images. We reduced the Spitzer photometry using the techniques of Nikolov et al. (2015), Sing et al. (2015, 2016), and Alam et al. (2018). We filtered for outliers in the data and subtracted the sky background using an iterative $3 \sigma$ clipping procedure, as outlined in Knutson et al. (2012) and Todorov et al. (2013).

We extracted photometric points using two approaches: fixed and time-variable aperture photometry. In the fixed approach, we used circular apertures with radii ranging from 4 to 8 pixels in increments of 0.5 . For the time-variable approach, we scaled the size of the aperture by the noise pixel parameter (the normalized effective background area of the IRAC point response function), which depends on the FWHM of the stellar PSF squared (Mighell 2005; Knutson et al. 2012; Lewis et al. 2013; Nikolov et al. 2015). We compared the light-curve residuals and the white and red noise components measured with the Carter \& Winn (2009) wavelet technique to identify the best results from both methods.

\subsection{Unusable WFC3 Observations}

We also observed one transit of WASP-62b with Hubble/ WFC3 on UT 2017 April 14 (GO: 14767). This visit, however, suffered from severe guide star issues that impacted the data quality of the observations, rendering it unsuitable for reliably extracting information on the planet's atmosphere. Guide star issues during the planet's transit impacted the telescope guiding, resulting in large drifts on the order of several pixels that affect the photometric quality of the spectroscopic channels and contribute to systematic trends that drift in wavelength during the observation (see Figure 11 of Sing 2018). Further, the G141 stellar spectra exhibit large offsets in the stellar spectral structure, even though the edges of the spectrum are sharp, likely due to warping of the scan as the scan rate changes across the detector as well as positional shifts in the scan. ${ }^{14}$ Although a spectrum extracted from this observation was presented in Skaf et al. (2020), we do not use this G141 data set in our analysis for the reasons outlined above.

\subsection{Host Star X-Ray and UV Monitoring}

We were awarded XMM-Newton time (program ID 80479 , P.I. J. Sanz-Forcada) to observe WASP-62 on UT 2017 April 14 for $\sim 8 \mathrm{ks}$. We fit European Photon Imaging Camera (EPIC) spectra using a one-temperature coronal model to calculate an X-ray luminosity $(0.12-2.48 \mathrm{keV})$ of $L_{\mathrm{X}}=$ $6.0 \times 10^{28} \mathrm{erg} \mathrm{s}^{-1}(\mathrm{~S} / \mathrm{N}=5.8)$, for a Gaia DR2 distance of $176.53 \pm 0.41 \mathrm{pc}$. The resulting $\log L_{\mathrm{X}} / L_{\mathrm{bol}}=-5.1$ measurement indicates that the star has a moderate activity level, similar to the young solar analog $\iota$ Hor (G0V) in which $\mathrm{X}$-ray variability is present but flares are not frequently observed (Sanz-Forcada et al. 2019).

Furthermore, the X-ray and UV light curves taken with the XMM-Newton EPIC $(\sim 1-124 \AA)$ and the Optical Monitor

\footnotetext{
${ }^{14}$ The reduced WFC3 stellar spectra are provided as supplementary material via figshare:10.6084/m9.figshare.13231904.v3.
} 
(OM)/UVM2 filter (2070-2550 $)$ show some level of variability. ${ }^{15}$ The UV light curve shows some dips (up to $\sim 10 \%$ absorption) around the beginning of the primary transit that could indicate the presence of occulted chromospheric plages as the planet transits. (Further details will be included in J. Sanz-Forcada et al. 2021, in preparation). Considering the activity level of the host star, we therefore account for starspots and faculae in our retrieval analysis (Section 4).

\section{Hubble and Spitzer Light-curve Fits}

\subsection{Hubble}

We fit the light curves following the procedure detailed in Kirk et al. (2017, 2018, 2019), which we briefly describe here. We modeled the analytic transit light curves of Mandel \& Agol (2002) using the Batman package (Kreidberg 2015), combined with a Gaussian process (GP) implemented with the george (Ambikasaran et al. 2015) code to model noise in the data. GPs are increasingly used in transmission spectroscopy (e.g., Gibson et al. 2012a, 2012b; Evans et al. 2015, 2017; Kirk et al. 2017, 2018, 2019; Louden et al. 2017; Parviainen et al. 2018).

For both the white light and spectroscopic light-curve fits, we used the four parameter nonlinear limb darkening law (Claret 2000). We derived the limb darkening coefficients using 3D stellar models (Magic et al. 2015), and fixed them to the theoretical values in our fits. For the white light-curve fits, we fixed the inclination $i$ to $88^{\circ} .3$, the scaled semimajor axis $a / R_{\star}$ to 9.53 , and the period $P$ to 4.4 days (based on the literature values from Hellier et al. 2012), and fit for the time of midtransit $T_{0}$, planet-to-star radius ratio $R_{p} / R_{\star}$, and the GP hyperparameters. Our GP was defined by the combination of 11 squared exponential kernels, each operating on a jitter detrending vector. ${ }^{16}$ These 11 kernels each had their own length scale but shared a common amplitude. We additionally included a white noise kernel, defined by the variance $\left(\sigma^{2}\right)$, to account for white noise unaccounted by the photometric error bars. The GP therefore added an additional 13 free parameters for each light curve.

Following similar studies (e.g., Evans et al. 2017, 2018), we standardized each GP input variable (jitter detrending variable) by subtracting the mean and dividing by the standard deviation. This method gives each standardized variable a mean of zero and a standard deviation of unity, which helps the GP to determine the inputs of importance for describing the noise characteristics. We fit for the natural log of the inverse length scale (e.g., Evans et al. 2017, 2018; Gibson et al. 2017). We placed wide, truncated uniform priors in log space on each of the hyperparameters. This choice assures uninformative priors since a uniform prior in log-space is akin to fitting for a $1 / \mathrm{x}$ prior in nonlogarithmic space. Since we are fitting for the natural $\log$ of the inverse length scale, this choice encourages the length scale to longer length scales so as to ensure that the GP does not over-fit the data (e.g., Evans et al. 2018; Parviainen et al. 2018; Gibson et al. 2019). The GP amplitude was bounded between 0.01 and $100 \times$ the variance of the outof-transit data, and the length scales were bounded by the

\footnotetext{
15 The stellar UV monitoring data is provided as supplementary material via figshare:doi:10.6084/m9.figshare.13231994.v2.

16 As described in Sing et al. (2019), the jitter vectors found to highly correlate with Hubble/STIS data are the right ascension and declination of the aperture reference as well as the roll of the telescope along the V2 and V3 axes.
}

typical spacing between data points and $5 \times$ the maximum length scale. The white noise variance was bounded between $10^{-10}$ and $25 \mathrm{ppm}$.

We ran a Markov Chain Monte Carlo (MCMC) using the emcee Python package (Foreman-Mackey et al. 2013) to perform the fitting. For all of the light-curve fits, we began by optimizing the GP hyperparameters to the out-of-transit data to find the starting locations for the GP hyperparameters. The starting value for $R_{p} / R_{\star}$ was taken from Hellier et al. (2012) and from visual inspection of the light curve for $T_{0}$. The chains were then initialized with a small scatter around these starting values. We ran the MCMC for 2000 steps with 300 walkers $\left(20 \times n_{p}\right.$, where $n_{p}$ is the number of parameters) and calculated the 16th, 50th, and 84th percentiles for each parameter after discarding the first 1000 steps as burn in. Following the george documentation $^{17}$, we then ran a second chain with the walkers initiated with a small scatter around the 50th percentile values for another 2000 steps with 300 walkers and again discarded the first 1000 steps. We measure $R_{p} / R_{\star}$ values of $0.11176_{-0.001394}^{+0.001374}$, $0.111961_{-0.000729}^{+0.00084}$, and $0.113189_{-0.000692}^{+0.000750}$, for visits 57,58 , and 59 , respectively, from the white light curves (Figure 1).

To derive the spectroscopic light curves, we binned the G430L and G750L spectra into 12 and 17 spectrophotometric channels, which are listed in the first column of Table 1 . We then fit and detrended each spectrophotometric light curve following the same procedure as the white light curves, but kept $T_{0}$ fixed to the result from the white light-curve fit. We ran MCMCs to each light curve, following the same process as for the white light curves but with 280 walkers since there was one fewer fit parameter. The resulting $R_{p} / R_{\star}$ values for each spectroscopic channel are presented in Table $1 .{ }^{18}$ We used the values of $R_{p} / R_{\star}$ given in Table 1 to derive the optical transmission spectrum shown in Figure 2.

\subsection{Spitzer}

We fit the $3.6 \mu \mathrm{m}$ and $4.5 \mu \mathrm{m}$ IRAC light curves following the methods described in Alam et al. (2018). Briefly, we corrected for flux variations from intra-pixel sensitivity (e.g., Charbonneau et al. 2005, 2008; Reach et al. 2005; Knutson et al. 2008; Ingalls et al. 2012; Krick et al. 2016), and accounted for systematics by fitting a model of the functional form:

$$
F(t)=c_{0}+c_{1} x+c_{2} x^{2}+c_{3} y+c_{4} y^{2}+c_{5} x y+c_{6} t,
$$

where $F(t)$ is the stellar flux as a function of time, the coefficients $c_{0}$ through $c_{6}$ are free parameters, $x$ and $y$ are the stellar centroid positions on the detector, and $t$ is time. We marginalized over all possible combinations of this model using the Gibson (2014) procedure. To measure $R_{p} / R_{\star}$ for the transmission spectrum, we fixed $P, a / R_{\star}$, and $i$ to the values from Hellier et al. (2012) and fit for $R_{p} / R_{\star}$ and $T_{0}$. The limb darkening coefficients were also fixed to their theoretical values based on 3D stellar atmosphere models. The measured $R_{p} / R_{\star}$ values are included in Table $1 .{ }^{19}$ We note that our transit depth measurements are slightly lower than those previously

\footnotetext{
${ }^{17}$ https://george.readthedocs.io/en/latest/

18 Figures of the white light curves and spectroscopic light curves are provided as supplementary material via figshare:doi:10.6084/m9.figshare.13231970.v1.

19 Figures of the fitted light curves are provided as supplementary material on figshare:doi:10.6084/m9.figshare.13232084.v2.
} 

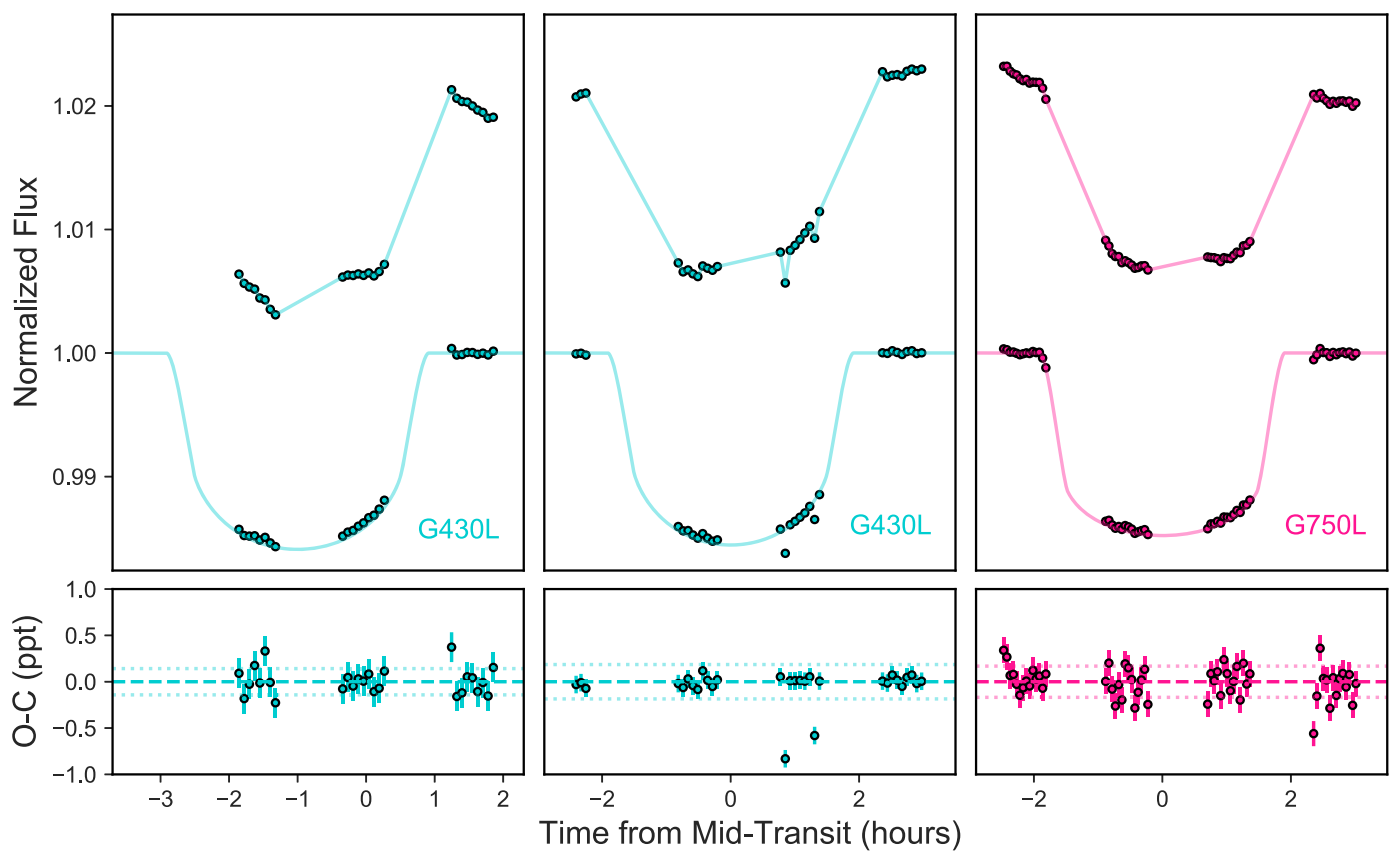

Figure 1. Top: the raw and detrended white light curves (excluding the first orbit and the first exposure of each subsequent orbit) for each HST visit in the STIS G430L (blue) and G750L (pink) gratings. The best-fit analytical light-curve model is overplotted. Bottom: transit fit residuals (in parts per thousand) with error bars.

Table 1

Transmission Spectrum of WASP-62b Measured with STIS G430L and G750L and Spitzer IRAC

\begin{tabular}{|c|c|}
\hline$\lambda(\AA)$ & $R_{p} / R_{*}$ \\
\hline $2900-3700$ & $0.11210_{-0.00115}^{+0.00116}$ \\
\hline $3700-4100$ & $0.11285_{-0.00066}^{+0.00072}$ \\
\hline $4100-4400$ & $0.11308_{-0.00051}^{+0.00053}$ \\
\hline $4400-4600$ & $0.11219_{-0.00053}^{+0.00054}$ \\
\hline $4600-4700$ & $0.11126_{-0.00053}^{+0.00050}$ \\
\hline $4700-4800$ & $0.11212_{-0.00044}^{+0.00043}$ \\
\hline $4800-4900$ & $0.11159_{-0.00049}^{+0.00053}$ \\
\hline $4900-5000$ & $0.11133_{-0.00067}^{+0.00070}$ \\
\hline $5000-5100$ & $0.11166_{-0.00059}^{+0.00055}$ \\
\hline $5100-5300$ & $0.11199_{-0.00039}^{+0.00042}$ \\
\hline $5300-5500$ & $0.11263_{-0.00063}^{+0.00052}$ \\
\hline $5500-5700$ & $0.11184_{-0.00049}^{+0.00049}$ \\
\hline $5700-5800$ & $0.11397_{-0.00078}^{+0.00069}$ \\
\hline $5800-5878$ & $0.11362_{-0.00068}^{+0.00066}$ \\
\hline $5878-5913$ & $0.11494_{-0.00066}^{+0.00063}$ \\
\hline 5913-6070 & $0.11290_{-0.00065}^{+0.00065}$ \\
\hline $6070-6200$ & $0.11227_{-0.00051}^{+0.00047}$ \\
\hline $6200-6300$ & $0.11299_{-0.00086}^{+0.00079}$ \\
\hline $6300-6513$ & $0.11376_{-0.00059}^{+0.00056}$ \\
\hline $6513-6613$ & $0.11489_{-0.00078}^{+0.00076}$ \\
\hline $6613-6800$ & $0.11208_{-0.00075}^{+0.00074}$ \\
\hline $6800-7000$ & $0.11344_{-0.00058}^{+0.00060}$ \\
\hline $7000-7200$ & $0.11130_{-0.00061}^{+0.00058}$ \\
\hline $7200-7590$ & $0.11077_{-0.00042}^{+0.00041}$ \\
\hline $7590-7740$ & $0.10910_{-0.00072}^{+0.00074}$ \\
\hline $7740-8100$ & $0.11136_{-0.00082}^{+0.00089}$ \\
\hline $8100-8500$ & $0.11346_{-0.00125}^{+0.00112}$ \\
\hline $8500-8985$ & $0.11417_{-0.00102}^{+0.00107}$ \\
\hline 8985-10300 & $0.11530_{-0.00154}^{+0.00152}$ \\
\hline 360000 & $0.10697 \pm 0.00152$ \\
\hline 450000 & $0.10962 \pm 0.00040$ \\
\hline
\end{tabular}

published in Garhart et al. (2020), and this discrepancy may be due to differences in the data reduction procedure, such as choice of baseline ramp shapes (linear versus quadratic) or where to trim out-of-transit data.

\section{Atmospheric Retrievals}

We retrieved the atmospheric properties of WASP-62b using the POSEIDON radiative transfer and retrieval code (MacDonald \& Madhusudhan 2017a). POSEIDON generates $\gtrsim 10^{5}-10^{6}$ model atmospheres - spanning a wide range of chemical abundances, temperature structures, and cloud properties - to identify the underlying atmospheric properties required to explain an observed exoplanet spectrum. Our initial exploration of WASP62b's STIS+Spitzer transmission spectrum considered 19 chemical species (Na, K, Li, H ${ }^{-}$, TiO, VO, $\mathrm{AlO}, \mathrm{SiO}, \mathrm{TiH}, \mathrm{CrH}, \mathrm{FeH}$, $\mathrm{CaH}, \mathrm{MgH}, \mathrm{NaH}, \mathrm{SiH}, \mathrm{H}_{2} \mathrm{O}, \mathrm{CH}_{4}, \mathrm{HCN}$, and $\mathrm{NH}_{3}$ ) known to present strong opacity at optical wavelengths (Tennyson \& Yurchenko 2018), collision-induced absorption due to $\mathrm{H}_{2}-\mathrm{H}_{2}$ and $\mathrm{H}_{2}-\mathrm{He}$ pairs (Karman et al. 2019), an isothermal temperature structure, and a patchy cloud/haze model (MacDonald \& Madhusudhan 2017a). Given the moderate stellar activity level indicated by the X-ray and UV photometric monitoring (Section 2.4), we also include a prescription for contamination due to unocculted starspots or faculae (Pinhas et al. 2018; Rackham et al. 2018). We explore this 28-dimensional parameter space with the Bayesian nested sampling algorithm MultiNest (Feroz \& Hobson 2008; Feroz et al. 2009, 2019), implemented by PyMultiNest (Buchner et al. 2014), with 4000 live points.

The best-fitting model from our atmospheric retrieval analysis is consistent with a clear atmosphere free from stellar contamination (Figure 2). Na I absorption, detected at 5.1 $\sigma$ confidence, characterizes WASP-62b's optical transmission spectrum. The observed pressure-broadened wings of the $\mathrm{Na}$ D-lines at $0.59 \mu \mathrm{m}$ strongly favor a clear atmosphere, permitting a precise retrieved $\mathrm{Na}$ abundance: $\log \left(\mathrm{X}_{\mathrm{Na}}\right)=-4.46_{-0.76}^{+1.09}$. The only other molecules with Bayes factors $>1$ are $\mathrm{H}_{2} \mathrm{O}, \mathrm{NH}_{3}, \mathrm{FeH}$, 

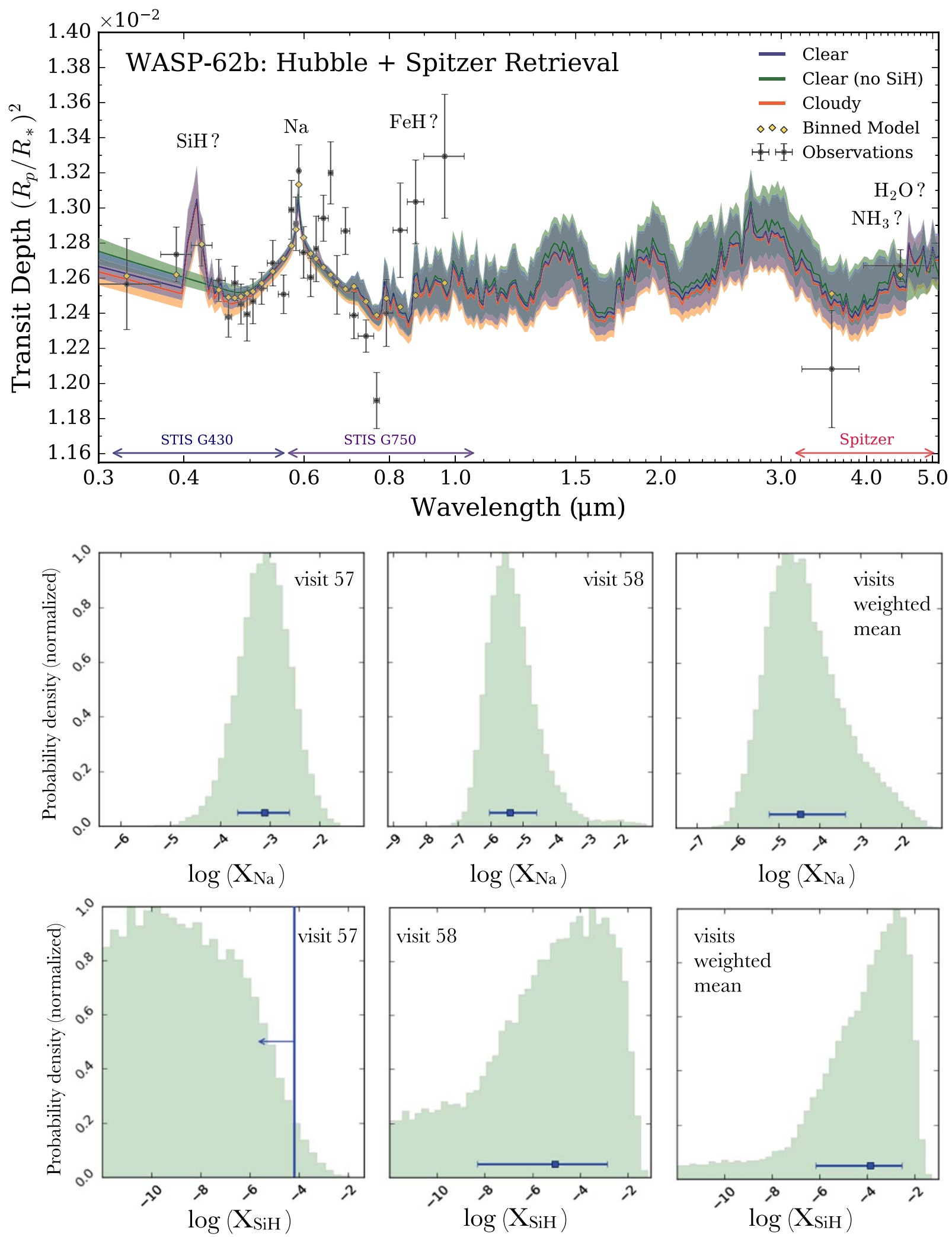

Figure 2. Top: transmission spectrum for WASP-62b measured with Hubble and Spitzer (black points), along with the best-fit model from our retrieval analysis (navy line) binned to the resolution of our observations (gold diamonds). The shaded regions indicate the $1 \sigma$ confidence intervals for the best-fit retrieved spectrum (blue), a clear model excluding $\mathrm{SiH}$ opacity (green), and a model incorporating clouds (orange). Middle: posterior histograms comparing the retrieved Na abundance when including only STIS G430L visit 57 (left), only G430L visit 58 (center), and the weighted mean of the two G430L data sets (right). Bottom: same as above, but for the retrieved $\mathrm{SiH}$ abundances.

and $\mathrm{SiH}$, with at least one of these species required at $2.8 \sigma$ confidence. We tentatively attribute a spectral feature around $0.4 \mu \mathrm{m}$ to $\mathrm{SiH}$ at $2.1 \sigma$ confidence.
The Bayesian evidences from models including clouds or stellar contamination were lower than the corresponding clear atmosphere models, indicating WASP-62b's observed transmission spectrum 
does not favor these features. We obtain a $2 \sigma$ lower limit on the cloud top pressure of $\log \left(P_{\text {cloud }}\right)>-5.56$. Based on this, we conclude that the observable atmosphere of WASP-62b is cloudfree at the pressures probed by our transmission spectra observations. We established, via successive Bayesian model comparisons, that the simplest model consistent with the present data is a clear, isothermal atmosphere with $\mathrm{Na}, \mathrm{H}_{2} \mathrm{O}, \mathrm{NH}_{3}, \mathrm{FeH}$, and $\mathrm{SiH}$. This seven-parameter model is compared to our STIS + Spitzer transmission spectrum observations in Figure 2.

The large scatter at the red end of the spectrum is responsible for the nonsignificant inference of $\mathrm{FeH}$. We tested how these spectrophotometric channels affect the retrieval results by repeating our analysis with the last three STIS G750L wavelength bins excluded. The removal of these three reddest STIS points changes the $\mathrm{FeH}$ posterior from a bounded constraint into an upper limit, but all other results remain consistent. We further note that an L-shaped degeneracy in the $\mathrm{H}_{2} \mathrm{O}-\mathrm{NH}_{3}$ correlation plot $^{20}$ suggests that the data require at least one of these species, but their absorption signatures are degenerate with the data in hand. The presence of $\mathrm{H}_{2} \mathrm{O}$ is indicated by the $4.5 \mu \mathrm{m}$ Spitzer channel, although further infrared observations are required to confirm $\mathrm{H}_{2} \mathrm{O}$ opacity and precisely constrain its abundance.

To investigate the reliability of our inferences, we also retrieved each STIS G430L visit separately (alongside the STIS G750L and Spitzer data). We compare the retrieved $\mathrm{Na}$ and $\mathrm{SiH}$ abundances from each G430L visit to our baseline two-visit weighted mean in the lower panels of Figure 2. Each visit separately detects the pressure-broadened $\mathrm{Na}$ wings, albeit with abundances discrepant by $\sim 2.5$ dex. In particular, the precise $\mathrm{Na}$ abundance from the visit 58 data set, $\log \left(\mathrm{X}_{\mathrm{Na}}\right)=-5.41_{-0.62}^{+0.82}$, is consistent with expectations for a solar metallicity atmosphere $\left(\log \left(\mathrm{X}_{\mathrm{Na}}\right)_{\odot}=-5.76\right.$, Asplund et al. 2009). The tentative evidence of $\mathrm{SiH}$ seems to be driven by visit 58, which is more precise (mean error $=139 \mathrm{ppm}$ ) than the visit 57 data set (483 ppm). However, the low abundance tail in the $\mathrm{SiH}$ posterior becomes less probable for the weighted mean of the two visits compared to visit 58 alone. This sharper $\mathrm{SiH}$ posterior for the combined visit retrieval suggests the spectra from each visit are not in tension, with the visit 57 retrievals not inferring $\mathrm{SiH}$ only due to the larger uncertainties.

We note that our retrieved limb temperature for WASP-62b, $835_{-150}^{+188} \mathrm{~K}$, is markedly lower than both the equilibrium temperature $\left(1394_{-20}^{+25} \mathrm{~K}\right)$ and skin temperature $(\sim 1170 \mathrm{~K})$ of the planet. This low retrieved temperature is consistent with the recent results of MacDonald et al. (2020), who showed that applying 1D atmospheric models to transmission spectra with different morning-evening terminator compositions leads to cooler retrieved temperatures. We additionally validated the isothermal assumption for the present data by running a retrieval with the six-parameter pressure-temperature profile of Madhusudhan \& Seager (2009). The results are consistent with those above, with only a marginal preference for a weak vertical temperature gradient at the terminator (Bayes factor $=1.4$ ).

For a further test, we ran self-consistent retrievals using the ATMO Retrieval Code (ARC) under the assumptions of

\footnotetext{
${ }^{20}$ Posterior distributions of retrieved parameters, and the transmission contribution function (Mollière et al. 2019) for our best-fitting model, are available as supplementary material on figshare:doi:10.6084/m9.fig share.13232252.v2 (retrieved parameters); doi:10.6084/m9.figshare.13232240. v2 (simulated retrieved parameters);doi:10.6084/m9.figshare.13232237.v3 (full retrieved parameters); doi:10.6084/m9.figshare.13232228.v2 (transmission contribution function).
}

chemical equilibrium and local condensation (e.g., Evans et al. 2017; Lewis et al. 2020). We ran one model including $\mathrm{K}$ and another model with $\mathrm{K}$ artificially removed (since the $\mathrm{K}$ feature is not observed in our data). Compared to the POSEIDON free retrieval results presented above, the self-consistent model finds somewhat hotter retrieved temperatures $\left(1069_{-48}^{+85} \mathrm{~K}\right.$ without potassium; $1059_{-44}^{+100} \mathrm{~K}$ with potassium) that are closer to the planet's equilibrium temperature $(1400 \mathrm{~K})$. These are, however, consistent with the retrieved temperature from the free retrieval to within $1 \sigma$.

The fit quality of the self-consistent model is strongly dependent on whether $\mathrm{K}$ absorption is included. Without $\mathrm{K}$ we find $\chi^{2}=79.74$, while this degrades to $\chi^{2}=99.57$ when $\mathrm{K}$ is included. This discrepancy suggests that some other process (besides local condensation) may be removing $\mathrm{K}$ from the gas phase. We note that the minimum $\chi^{2}$ somewhat prefers our minimal (seven-parameter) free retrieval $\left(\chi^{2}=60.48\right)$ over the self-consistent retrievals.

\section{Discussion \\ 5.1. A Clear Atmosphere for WASP-62b}

Due to the nearly ubiquitous nature of condensation clouds and photochemical hazes in exoplanet atmospheres (e.g., Wakeford et al. 2019), few benchmark planets with cloud-free, haze-free atmospheres at the pressures probed by transmission spectroscopy are currently known. The detection of the $\mathrm{Na} I$ line wings at $0.59 \mu \mathrm{m}$ in the atmosphere of WASP-62b marks the first spacebased observation of the pressure-broadened wings of the $\mathrm{Na}$ D-lines, and suggests that WASP-62b possesses a clear terminator. Clear atmosphere exoplanets present an unmatched opportunity to obtain increasingly precise retrieved abundance constraints, since they are unhampered by cloud-composition degeneracies (e.g., Figure 10 of MacDonald \& Madhusudhan 2017a).

We compare our results for WASP-62b with the groundbased VLT/FORS2 observations of WASP-96b, the clearest known exoplanet to date (Nikolov et al. 2018), in Figure 3. Although both WASP-62b and WASP-96b display the pressure-broadened $\mathrm{Na}$ line wings, WASP-62b shows no evidence of K I absorption at $0.76 \mu \mathrm{m}$ whereas WASP-96b is consistent with weak evidence of K I and Li I (Nikolov et al. 2018). The missing or weak potassium absorption for these clear planets is contrary to atmospheric models, which predict the presence of both alkali features in clear exoplanets (Seager \& Sasselov 2000). This observed trend may be due to a difference in the primordial abundances of the species, since $\mathrm{Na}$ is $\sim 15$ times more abundant than $\mathrm{K}$ in a solar abundance atmosphere (Lodders 2003). The relative condensation temperatures of $\mathrm{NaCl}$ and $\mathrm{KCl}$, as well as the photoionization energies of sodium and potassium, may further alter the relative amplitudes of the $\mathrm{Na}$ and $\mathrm{K}$ absorption features (Nikolov et al. 2018).

\subsection{ATMO Predictions for Si-bearing Species}

In light of our tentative inference of $\mathrm{SiH}$ opacity in WASP62b's transmission spectrum, here we consider theoretical predictions for gas-phase Si-bearing molecules at the equilibrium temperature $\left(T_{\text {eq }}=1440 \pm 30 \mathrm{~K}\right.$; Hellier et al. 2012) of WASP-62b. Equilibrium chemistry expectations for silicates suggest that the dominant $\mathrm{Si}$-bearing species are $\mathrm{SiO}$ and $\mathrm{SiS}$ at $\sim 1400 \mathrm{~K}$ (Visscher et al. 2010; Woitke et al. 2018). We investigate predictions for Si-bearing species in the atmosphere 


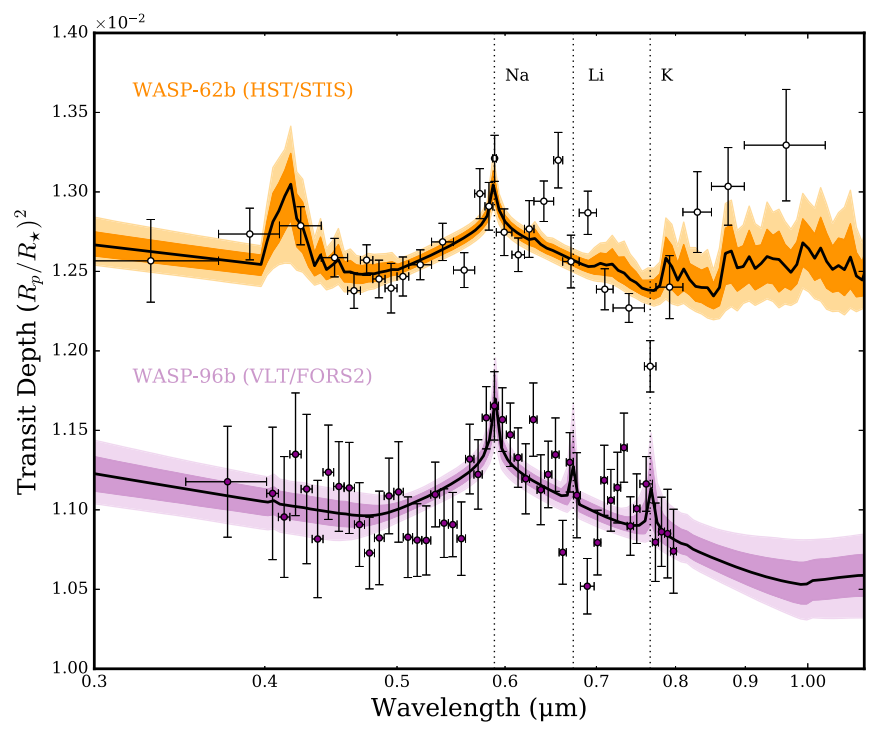

Figure 3. The Hubble/STIS optical transmission spectra (white points) of WASP-62b in this work, compared with the VLT/FORS2 transmission spectrum (purple points) of WASP-96b (Nikolov et al. 2018; offset vertically for clarity). The shaded regions show the $1 \sigma$ and $2 \sigma$ confidence intervals from cloud-free POSEIDON retrieval models for each planet. At low spectral resolution, both data sets display the prominent pressure-broadened wings of the $\mathrm{Na}$ D-lines at $0.59 \mu \mathrm{m}$. WASP-96b additionally displays weak evidence of $\mathrm{K} \mathrm{I}$ at $0.77 \mu \mathrm{m}$ and a hint of $\mathrm{Li} \mathrm{I}$ at $0.67 \mu \mathrm{m}$. Our transmission spectrum of WASP-62b represents the first clear detection of pressure-broadened $\mathrm{Na}$ wings from a space-based telescope.

of WASP-62b using the planet-specific forward model ATMO grid (e.g., Tremblin et al. 2015; Goyal et al. 2020) of 1D radiative-convective equilibrium pressure-temperature profiles with corresponding self-consistent equilibrium chemistry. This grid of model atmospheres spans a range of recirculation factors (RCF), metallicities, and $\mathrm{C} / \mathrm{O}$ ratios. $^{21}$ The $\mathrm{RCF}$ parameterizes the redistribution of input stellar energy in the planetary atmosphere, where a value of 0.5 represents efficient redistribution and 1 corresponds to no redistribution.

Figure 4 (top panel) shows the equilibrium chemical abundances of $\mathrm{H}_{2} \mathrm{O}, \mathrm{SiH}, \mathrm{SiO}$, and $\mathrm{SiS}$ with uniform redistribution $(\mathrm{RCF}=0.5)$, solar metallicity, and a solar $\mathrm{C} / \mathrm{O}$ ratio. While $\mathrm{SiH}$ is tentatively inferred by our retrievals, equilibrium expectations predict that $\mathrm{SiO}$ and $\mathrm{SiS}$ should be orders of magnitude more abundant than $\mathrm{SiH}$ in WASP-62b's atmosphere. Since silicate condensation has also been found to increase the carbon-tooxygen $(\mathrm{C} / \mathrm{O})$ ratio in gas from solar to super-solar values (Woitke et al. 2018), we also investigate how the abundances of these species change for sub-solar, solar, and super-solar $\mathrm{C} / \mathrm{O}$. Figure 4 (bottom panel) shows that $\mathrm{SiS}$ overtakes $\mathrm{SiO}$ to become the most abundant Si-bearing molecule for $\mathrm{C} / \mathrm{O} \gtrsim 0.7$. This is driven by a decrease in the $\mathrm{SiO}$ abundance for super-solar $\mathrm{C} / \mathrm{O}$ ratios, analogous to the well-known decrease in $\mathrm{H}_{2} \mathrm{O}$ abundance for enhanced $\mathrm{C} / \mathrm{O}$ ratios (Madhusudhan 2012). These results expand upon the silicon chemistry predictions from Visscher et al. (2010) — who considered rainout $\mathrm{Si}$ chemistry at a solar C/O ratio - demonstrating that $\mathrm{SiS}$ may be the most abundant gas-phase $\mathrm{Si}$ bearing species for exoplanets with super-solar $\mathrm{C} / \mathrm{O}$ ratios.

These equilibrium predictions appear in tension with our inferred $\mathrm{SiH}$ abundance $\left(\sim 10^{-4}\right)$. Our retrievals do not infer

\footnotetext{
21 We produced a range of $\mathrm{C} / \mathrm{O}$ ratios by varying the oxygen abundance $\mathrm{O} / \mathrm{H}$, as detailed in Sections 4 and 2.3 of the supplementary material of Goyal et al. (2020).
}
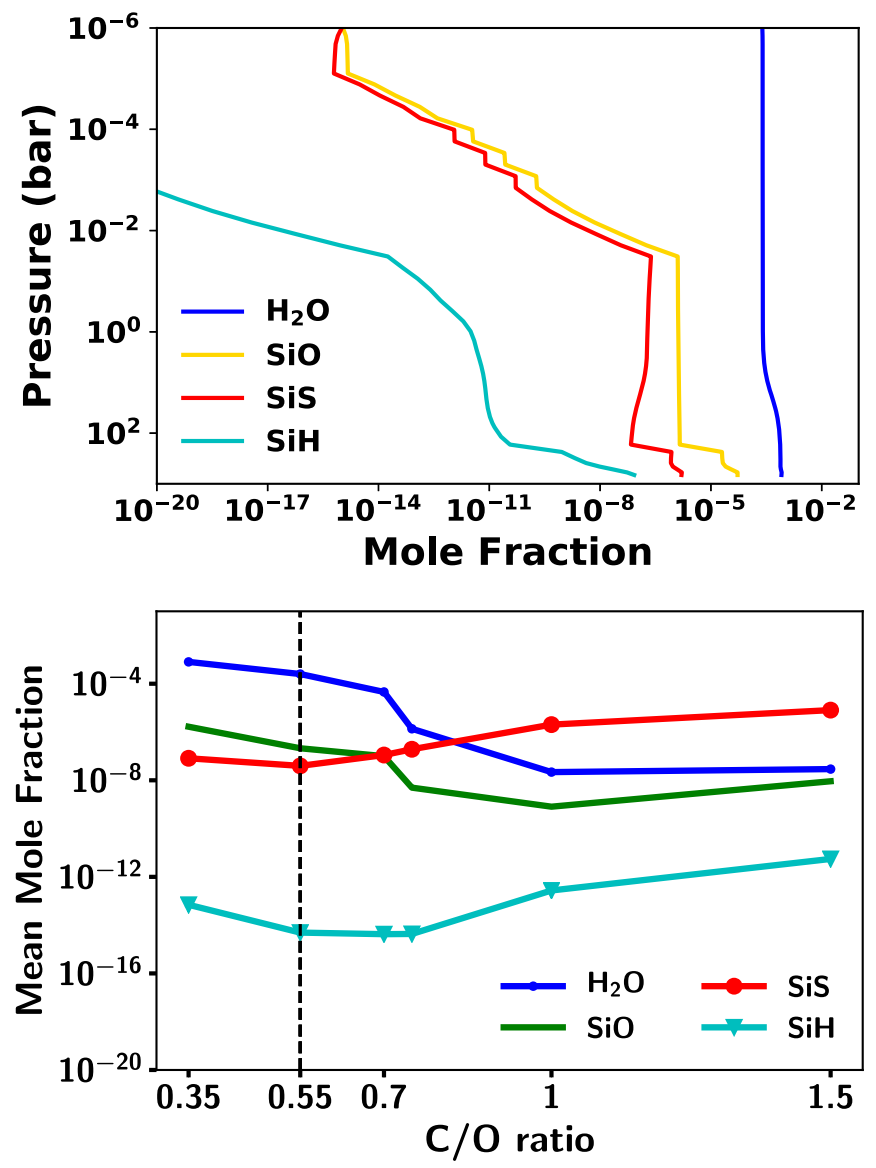

Figure 4. Top: theoretical abundances of $\mathrm{H}_{2} \mathrm{O}$ (blue), $\mathrm{SiO}$ (yellow), $\mathrm{SiS}$ (red), and $\mathrm{SiH}$ (cyan) based on 1D equilibrium chemistry ATMO models of WASP$62 \mathrm{~b}$ at solar metallicity. $\mathrm{SiH}$ is predicted to be less abundant than $\mathrm{SiS}$ or $\mathrm{SiO}$ at the equilibrium temperature of WASP-62b. Bottom: mean abundances (between 0.1 and 100 mbar) for these molecules as $\mathrm{C} / \mathrm{O}$ varies. The vertical dashed line denotes a solar $\mathrm{C} / \mathrm{O}$. The abundances of $\mathrm{SiO}$ and $\mathrm{H}_{2} \mathrm{O}$ drop by orders of magnitude for even moderately super-solar $\mathrm{C} / \mathrm{O}$, with $\mathrm{SiS}$ predicted to be the dominant Si-bearing molecule for $\mathrm{C} / \mathrm{O} \gtrsim 0.7$.

$\mathrm{SiO}$, despite its inclusion, due to its characteristic slope at UVoptical wavelengths (e.g. Sharp \& Burrows 2007) differing from the feature at $0.4 \mu \mathrm{m}$ we attribute to $\mathrm{SiH}$. Evidence of SiS cannot currently be assessed at optical wavelengths, due to the lack of currently available line lists for low energy (bluer than $\sim 2 \mu \mathrm{m}$ ) transitions (Upadhyay et al. 2018). It is therefore possible that the spectral feature we attribute to $\mathrm{SiH}$ could be a misclassified SiS feature-optical line list data for SiS would resolve this ambiguity. However, we stress that the evidence for $\mathrm{SiH}$ from our STIS data remains low $(2.1 \sigma)$, and future observations will be required to assess the presence of gasphase Si-bearing species in WASP-62b's atmosphere.

\subsection{Predictions for JWST}

A clear atmosphere for WASP-62b opens the door to extreme precision molecular abundance measurements from early JWST observations. To quantitatively assess the potential of WASP-62b as a priority JWST target, we offer predictions from a simulated retrieval analysis of synthetic JWST ERS observations of WASP-62b.

We generated a moderate-resolution $(R=10,000)$ model transmission spectrum, including $\mathrm{Na}, \mathrm{H}_{2} \mathrm{O}, \mathrm{NH}_{3}, \mathrm{FeH}$, and $\mathrm{SiH}$, with the median retrieved atmospheric properties from our 

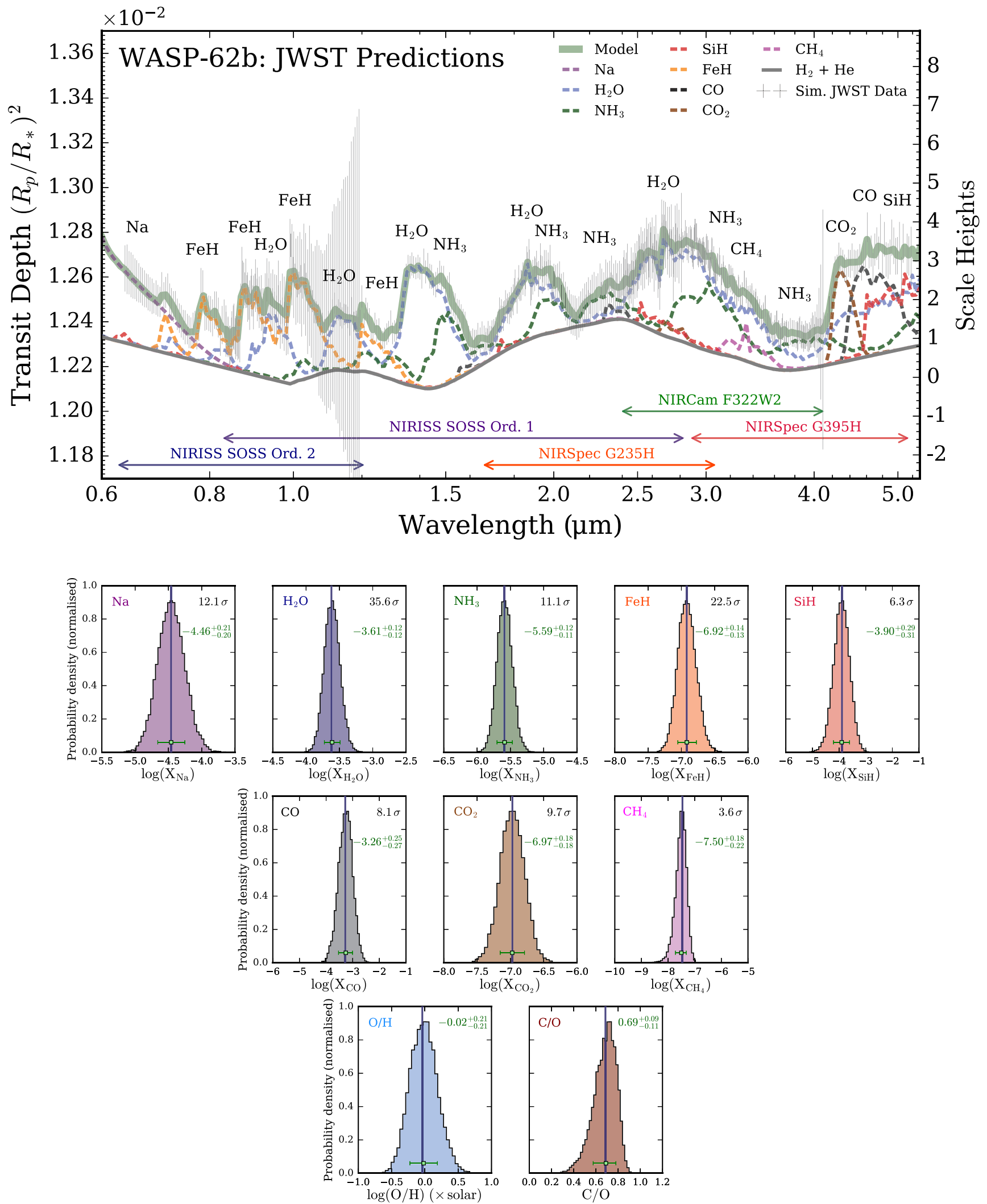

Figure 5. Top: simulated JWST observations for NIRSpec G235H+G395H, NIRCam F322W2, and NIRISS SOSS orders 1 and 2 modes (gray points), for a single transit of WASP-62b with each mode, along with the best-fitting retrieved model (solid green line) and associated opacity contributions from $\mathrm{Na}_{2} \mathrm{H}_{2} \mathrm{O}, \mathrm{NH}_{3}, \mathrm{SiH}_{\text {, }}$ $\mathrm{FeH}, \mathrm{CO}, \mathrm{CO}_{2}$, and $\mathrm{CH}_{4}$ (dashed lines), and the $\mathrm{H}_{2}+\mathrm{He}$ continuum (solid gray line). Bottom: retrieved abundance posteriors for $\mathrm{Na}, \mathrm{H}_{2} \mathrm{O}, \mathrm{NH} 3, \mathrm{FeH}, \mathrm{SiH}, \mathrm{CO}, \mathrm{CO} 2$, $\mathrm{CH}_{4}$, metallicity $(\mathrm{O} / \mathrm{H})$, and $\mathrm{C} / \mathrm{O}$. The abundances used to generate the model (solid vertical lines) agree well with the retrieved abundances (green error bars). JWST can conclusively detect and obtain precise abundance constraints for all the optical and infrared absorbers included in our model within the scope of the JWST ERS program. 
HST+Spitzer analysis. To explore the ability of JWST to constrain WASP-62's $\mathrm{C} / \mathrm{O}$ ratio, we additionally injected $\mathrm{CO}$, $\mathrm{CO}_{2}$, and $\mathrm{CH}_{4}$ abundances into our model. The representative values were taken to be the 10 mbar abundances of these species from the self-consistent model grid of Goyal et al. (2020), assumed uniform throughout the atmosphere. We then simulated JWST ERS observations with PandExo ${ }^{22}$ (Batalha et al. 2017) - in the Panchromatic Transmission configuration outlined by Bean et al. (2018)—spanning four transits across the following modes:

1. NIRISS SOSS orders 1 and $2(0.64-2.8 \mu \mathrm{m})[61 \mathrm{ppm} /$ $141 \mathrm{ppm}]$

2. NIRSpec G235H (1.7-3.1 $\mu \mathrm{m})$ [39 ppm].

3. NIRCam F322W2 $(2.4-4.1 \mu \mathrm{m})$ [67 ppm].

4. NIRSpec G395H (2.9-5.2 $\mu \mathrm{m})$ [57 ppm].

We binned the simulated PandExo observations to $R=100$ for all modes, neglecting errors exceeding $1000 \mathrm{ppm}$ (mainly the reddest NIRISS SOSS second-order data and near the NIRSpec detector gaps at $2.24 \mu \mathrm{m}$ and $3.80 \mu \mathrm{m}$ ), for a total of 677 data points. The square brackets above denote the resulting mean data precision for each mode. We removed Gaussian scatter from the data set, centering the data on the actual model, to ensure our retrieval results are unbiased by a given noise instance (see Feng et al. 2018). Finally, we retrieved the synthetic data set using POSEIDON.

Our simulated JWST observations and best-fitting retrieved model spectrum are shown in Figure 5. The combined ERS instrument modes can detect many prominent optical and infrared spectral features, as well as obtain precise constraints on the planetary atmospheric metallicity (here taken as $\mathrm{O} / \mathrm{H}$ ) and $\mathrm{C} / \mathrm{O}$. Each JWST ERS mode covers at least one of the $\mathrm{H}_{2} \mathrm{O}$ band heads at $0.95 \mu \mathrm{m}, 1.15 \mu \mathrm{m}, 1.4 \mu \mathrm{m}, 1.9 \mu \mathrm{m}, 2.7 \mu \mathrm{m}$, and $4.3 \mu \mathrm{m}$. NIRISS SOSS samples both the red wings of the $\mathrm{Na}$ D-lines and strong $\mathrm{FeH}$ features at $0.8 \mu \mathrm{m}, 0.9 \mu \mathrm{m}$, and $1.0 \mu \mathrm{m}$. Both NIRISS SOSS and NIRSpec G235H are highly sensitive to $\mathrm{NH}_{3}$ absorption, via the K-band feature at $2.2 \mu \mathrm{m}$ (see also MacDonald \& Madhusudhan 2017b). NIRCam F322W2 and NIRSpec G395H sample the $\mathrm{CO}, \mathrm{CO}_{2}$, and $\mathrm{CH}_{4}$ features between 3 and $5 \mu \mathrm{m}$. NIRSpec G395H can also probe a broad $\mathrm{SiH}$ feature from $\sim 4.6$ to $5.3 \mu \mathrm{m}$, testing if gasphase silicon species are indeed present in WASP-62b's atmosphere. The combined observing configuration also provides the optical baseline necessary for obtaining precise molecular abundances and atmospheric metallicities.

We predict that JWST observations of WASP-62b, within the scope of the ERS program, can conclusively detect $\mathrm{Na}$ $(12.1 \sigma), \mathrm{H}_{2} \mathrm{O}(35.6 \sigma), \mathrm{FeH}(22.5 \sigma), \mathrm{SiH}(6.3 \sigma), \mathrm{NH}_{3}(11.1 \sigma)$, $\mathrm{CO}(8.1 \sigma), \mathrm{CO}_{2}(9.7 \sigma)$, and $\mathrm{CH}_{4}(3.6 \sigma)$. The clear atmosphere offers remarkably precise abundance constraints: 0.12 dex for $\mathrm{H}_{2} \mathrm{O}, 0.14$ dex for $\mathrm{FeH}, 0.21$ dex for $\mathrm{Na}$, and 0.30 dex for $\mathrm{SiH}$, $0.26 \mathrm{dex}$ for $\mathrm{CO}, 0.18 \mathrm{dex}$ for $\mathrm{CO}_{2}$, and $0.20 \mathrm{dex}$ for $\mathrm{CH}_{4}$ (shown in Figure 5, lower panels). These predicted abundance constraints, from a single transit with each JWST mode, would immediately outclass the most precise abundances obtained for hot Jupiters with existing facilities to date $\left(\lesssim 0.3\right.$ dex for $\mathrm{H}_{2} \mathrm{O}$, e.g., Welbanks et al. 2019).

One of the key goals of JWST is to measure the metallicity and $\mathrm{C} / \mathrm{O}$ ratios of a population of exoplanets (Beichman et al. 2014; Bean et al. 2018). Our injected abundances of $\mathrm{CO}, \mathrm{CO}_{2}$,

\footnotetext{
${ }^{22}$ https://natashabatalha.github.io/PandExo/
}

and $\mathrm{CH}_{4}$ allow us to explore the expected constraints achievable by the JWST ERS program. We derive posterior distributions for the metallicity $(\mathrm{O} / \mathrm{H}$ in solar units) and $\mathrm{C} / \mathrm{O}$ from our individual abundance posteriors using the methodology of MacDonald \& Madhusudhan (2019), as shown on the bottom row of Figure 5 . We predict that it is possible to constrain WASP-62b's $\mathrm{C} / \mathrm{O}$ to \pm 0.1 and the atmospheric metallicity to \pm 0.21 dex. Note that these constraints do not require the assumption of chemical equilibrium.

\section{Summary and Conclusions}

We presented the STIS+Spitzer transmission spectrum of WASP-62b, the only transiting giant planet currently known in the JWST CVZ. WASP-62b is one of the few exoplanets with observed pressure-broadened alkali line wings, and the first space-based transmission spectrum displaying pressure-broadening $\mathrm{Na}$ I absorption. Our retrievals are consistent with a cloud-free, haze-free atmosphere, with a strong detection of $\mathrm{Na}$ I at $5.1 \sigma$ confidence and tentative evidence of $\mathrm{SiH}$ at $0.4 \mu \mathrm{m}$.

We explored the prospects for JWST observations of WASP$62 \mathrm{~b}$ via a simulated retrieval exercise. Conclusive detections $(>5 \sigma)$ of $\mathrm{Na}, \mathrm{H}_{2} \mathrm{O}, \mathrm{FeH}$, and $\mathrm{SiH}$ can be achieved within the scope of the JWST ERS program, with the clear nature of WASP-62b's atmosphere offering abundance constraints at $<0.2$ dex precision. If confirmed, gas-phase $\mathrm{SiH}$ features observed near $5 \mu \mathrm{m}$ would complement observations of condensed silicates (e.g., Gao et al. 2020) via vibrational mode resonance features - accessible with JWST's MIRI LRS mode at longer wavelengths (Wakeford \& Sing 2015). These results suggest WASP-62b is an exceptional target for JWST transmission spectroscopy.

In preparation for JWST, identifying targets that are cloudfree/haze-free is important for mobilizing community efforts to observe the best planets for detailed atmospheric follow-up. Although alternative targets have since been put forward, WASP-62 is the only star in the JWST CVZ with a known transiting giant planet that is bright enough for high-quality atmospheric characterization via transit spectroscopy. JWST transit programs require many repeated visits, which ideally could be scheduled at any time of the year and executed quickly. WASP-62b is therefore one of the most readily accessible targets for atmospheric studies with JWST.

The authors thank the referee for insightful comments and suggestions. M.K.A. thanks Mark Marley for useful discussions. R.J.M. thanks Sergei Yurchenko for helpful discussions on molecular line lists. This paper makes use of observations from the NASA/ESA Hubble Space Telescope, obtained at the Space Telescope Science Institute, which is operated by the Association of Universities for Research in Astronomy, Inc., under NASA contract NAS 5-26555. These observations are associated with the Hubble GO program 14767. M.K.A. acknowledges support by the National Science Foundation through a Graduate Research Fellowship. J.S.F. acknowledges support from the Spanish State Research Agency project AYA2016-79425-C3-2-P. Per AAS Journals policy, all supplementary figures are included in this public figshare repository.

Software: Batman (Kreidberg 2015), george (Ambikasaran et al. 2015), emcee (Foreman-Mackey et al. 2013), POSEIDON (MacDonald \& Madhusudhan 2017a). 


\section{ORCID iDs}

Munazza K. Alam (1) https://orcid.org/0000-0003-4157-832X Mercedes López-Morales (1) https://orcid.org/0000-00033204-8183

Ryan J. MacDonald 지 https://orcid.org/0000-0003-4816-3469

Nikolay Nikolov (1) https://orcid.org/0000-0002-6500-3574

James Kirk iㅣ https://orcid.org/0000-0002-4207-6615

Jayesh M. Goyal (10) https://orcid.org/0000-0002-8515-7204

David K. Sing iํ https://orcid.org/0000-0001-6050-7645

Hannah R. Wakeford (1) https://orcid.org/0000-00034328-3867

Alexander D. Rathcke (ib https://orcid.org/0000-00024227-4953

Drake L. Deming (1) https://orcid.org/0000-0001-5727-4094

Jorge Sanz-Forcada $\odot$ https://orcid.org/0000-0002-1600-7835

Nikole K. Lewis (1) https://orcid.org/0000-0002-8507-1304

Joanna K. Barstow (1) https://orcid.org/0000-0003-3726-5419

Thomas Mikal-Evans 주 https://orcid.org/0000-0001-

5442-1300

Lars A. Buchhave (i) https://orcid.org/0000-0003-1605-5666

\section{References}

Alam, M. K., Nikolov, N., López-Morales, M., et al. 2018, AJ, 156, 298 Alam, M. K., López-Morales, M., Nikolov, N., et al. 2020, AJ, 160, 51 Ambikasaran, S., Foreman-Mackey, D., Greengard, L., Hogg, D. W., \& O'Neil, M. 2015, ITPAM, 38, 252

Asplund, M., Grevesse, N., Sauval, A. J., \& Scott, P. 2009, ARA\&A, 47, 481 Batalha, N. E., Mandell, A., Pontoppidan, K., et al. 2017, PASP, 129, 064501 Bean, J. L., Stevenson, K. B., Batalha, N. M., et al. 2018, PASP, 130, 114402 Beichman, C., Benneke, B., Knutson, H., et al. 2014, PASP, 126, 1134 Buchner, J., Georgakakis, A., Nandra, K., et al. 2014, A\&A, 564, A125 Carter, J. A., \& Winn, J. N. 2009, ApJ, 704, 51

Charbonneau, D., Knutson, H. A., Barman, T., et al. 2008, ApJ, 686, 1341

Charbonneau, D., Allen, L. E., Megeath, S. T., et al. 2005, ApJ, 626, 523

Claret, A. 2000, A\&A, 363, 1081

Evans, T. M., Aigrain, S., Gibson, N., et al. 2015, MNRAS, 451, 680

Evans, T. M., Sing, D. K., Kataria, T., et al. 2017, Natur, 548, 58

Evans, T. M., Sing, D. K., Goyal, J. M., et al. 2018, AJ, 156, 283

Fazio, G. G., Hora, J. L., Allen, L. E., et al. 2004, ApJS, 154, 10

Feng, Y. K., Robinson, T. D., Fortney, J. J., et al. 2018, AJ, 155, 200

Feroz, F., \& Hobson, M. P. 2008, MNRAS, 384, 449

Feroz, F., Hobson, M. P., \& Bridges, M. 2009, MNRAS, 398, 1601

Feroz, F., Hobson, M. P., Cameron, E., \& Pettitt, A. N. 2019, OJAp, 2, 10

Fischer, P. D., Knutson, H. A., Sing, D. K., et al. 2016, ApJ, 827, 19

Foreman-Mackey, D., Hogg, D. W., Lang, D., \& Goodman, J. 2013, PASP, 125,306

Fraine, J., Deming, D., Benneke, B., et al. 2014, Natur, 513, 526

Gao, P., Thorngren, D. P., Lee, G. K. H., et al. 2020, NatAs, 4, 951

Garhart, E., Deming, D., Mandell, A., et al. 2020, AJ, 159, 137

Gibson, N. P. 2014, MNRAS, 445, 3401

Gibson, N. P., Aigrain, S., Roberts, S., et al. 2012a, MNRAS, 419, 2683

Gibson, N. P., de Mooij, E. J. W., Evans, T. M., et al. 2019, MNRAS, 482, 606

Gibson, N. P., Nikolov, N., Sing, D. K., et al. 2017, MNRAS, 467, 4591

Gibson, N. P., Aigrain, S., Pont, F., et al. 2012b, MNRAS, 422, 753

Goyal, J. M., Mayne, N., Drummond, B., et al. 2020, MNRAS, 498, 4680

Hellier, C., Anderson, D. R., Collier Cameron, A., et al. 2012, MNRAS, 426, 739
Huitson, C. M., Sing, D. K., Pont, F., et al. 2013, MNRAS, 434, 3252 Ingalls, J. G., Krick, J. E., Carey, S. J., et al. 2012, Proc. SPIE, 8442, 84421Y Karman, T., Gordon, I. E., van der Avoird, A., et al. 2019, Icar, 328, 160 Kilpatrick, B. M., Cubillos, P. E., Stevenson, K. B., et al. 2018, AJ, 156, 103 Kirk, J., López-Morales, M., Wheatley, P. J., et al. 2019, AJ, 158, 144 Kirk, J., Wheatley, P. J., Louden, T., et al. 2017, MNRAS, 468, 3907 Kirk, J., Wheatley, P. J., Louden, T., et al. 2018, MNRAS, 474, 876 Knutson, H. A., Charbonneau, D., Allen, L. E., Burrows, A., \& Megeath, S. T. 2008, ApJ, 673, 526

Knutson, H. A., Lewis, N., Fortney, J. J., et al. 2012, ApJ, 754, 22 Kreidberg, L. 2015, PASP, 127, 1161

Kreidberg, L., Bean, J. L., Désert, J.-M., et al. 2014, ApJL, 793, L27

Krick, J. E., Ingalls, J., Carey, S., et al. 2016, ApJ, 824, 27

Lewis, N. K., Knutson, H. A., Showman, A. P., et al. 2013, ApJ, 766, 95

Lewis, N. K., Wakeford, H. R., MacDonald, R. J., et al. 2020, ApJ, 902, 19

Lodders, K. 2003, ApJ, 591, 1220

Louden, T., Wheatley, P. J., Irwin, P. G. J., Kirk, J., \& Skillen, I. 2017 , MNRAS, 470, 742

MacDonald, R. J., Goyal, J. M., \& Lewis, N. K. 2020, ApJL, 893, L43

MacDonald, R. J., \& Madhusudhan, N. 2017a, MNRAS, 469, 1979

MacDonald, R. J., \& Madhusudhan, N. 2017b, ApJL, 850, L15

MacDonald, R. J., \& Madhusudhan, N. 2019, MNRAS, 486, 1292

Madhusudhan, N. 2012, ApJ, 758, 36

Madhusudhan, N., \& Seager, S. 2009, ApJ, 707, 24

Magic, Z., Chiavassa, A., Collet, R., \& Asplund, M. 2015, A\&A, 573, A90

Mandel, K., \& Agol, E. 2002, ApJL, 580, L171

Mighell, K. J. 2005, MNRAS, 361, 861

Mollière, P., Wardenier, J. P., van Boekel, R., et al. 2019, A\&A, 627, A67

Mordasini, C., van Boekel, R., Mollière, P., Henning, T., \& Benneke, B. 2016, ApJ, 832, 41

Nikolov, N., Sing, D. K., Gibson, N. P., et al. 2016, ApJ, 832, 191

Nikolov, N., Sing, D. K., Burrows, A. S., et al. 2015, MNRAS, 447, 463

Nikolov, N., Sing, D. K., Fortney, J. J., et al. 2018, Natur, 557, 526

Öberg, K. I., Murray-Clay, R., \& Bergin, E. A. 2011, ApJL, 743, L16

Parviainen, H., Pallé, E., Chen, G., et al. 2018, A\&A, 609, A33

Pinhas, A., Rackham, B. V., Madhusudhan, N., \& Apai, D. 2018, MNRAS, 480, 5314

Rackham, B. V., Apai, D., \& Giampapa, M. S. 2018, ApJ, 853, 122

Reach, W. T., Megeath, S. T., Cohen, M., et al. 2005, PASP, 117, 978

Sanz-Forcada, J., Stelzer, B., Coffaro, M., Raetz, S., \& Alvarado-Gómez, J. D. 2019, A\&A, 631, A45

Seager, S., \& Sasselov, D. D. 2000, ApJ, 537, 916

Sharp, C. M., \& Burrows, A. 2007, ApJS, 168, 140

Sing, D. K. 2018, arXiv:1804.07357

Sing, D. K., Wakeford, H. R., Showman, A. P., et al. 2015, MNRAS, 446, 2428

Sing, D. K., Fortney, J. J., Nikolov, N., et al. 2016, Natur, 529, 59

Sing, D. K., Lavvas, P., Ballester, G. E., et al. 2019, AJ, 158, 91

Skaf, N., Fabienne Bieger, M., Edwards, B., et al. 2020, AJ, 160, 109

Stevenson, K. B., Lewis, N. K., Bean, J. L., et al. 2016, PASP, 128, 094401

Tennyson, J., \& Yurchenko, S. 2018, Atoms, 6, 26

Todorov, K. O., Deming, D., Knutson, H. A., et al. 2013, ApJ, 770, 102

Tremblin, P., Amundsen, D. S., Mourier, P., et al. 2015, ApJL, 804, L17

Upadhyay, A., Conway, E. K., Tennyson, J., \& Yurchenko, S. N. 2018, MNRAS, 477, 1520

Visscher, C., Lodders, K., \& Fegley, B. J. 2010, ApJ, 716, 1060

Wakeford, H. R., \& Sing, D. K. 2015, A\&A, 573, A122

Wakeford, H. R., Wilson, T. J., Stevenson, K. B., \& Lewis, N. K. 2019, RNAAS, 3, 7

Wakeford, H. R., Sing, D. K., Deming, D., et al. 2018, AJ, 155, 29

Welbanks, L., Madhusudhan, N., Allard, N. F., et al. 2019, ApJL, 887, L20

Werner, M. W., Roellig, T. L., Low, F. J., et al. 2004, ApJS, 154, 1

Woitke, P., Helling, C., Hunter, G. H., et al. 2018, A\&A, 614, A1 